\title{
Determinación electroquímica cuantitativa de la velocidad de corrosión de un acero de construcción. Evaluación de la susceptibilidad al ataque por picaduras

\author{
Parte 1I: Ensayos en mortero
} \\ C. ANDRADE I. I. A. CONZIIIEZ (*)
}

\section{INTRODUCCION}

El estudio del efecto de un determinado aditivo sobre la corrosión de las armaduras, o el de un hormigón con fisuras, o sometido a ambientes marinos o, en fin, de cualquier factor o circunstancia calificada como agresiva, suele exigir un laborioso y extenso programa de trabajo que contemple todas las posibilidades por las que puedan pasar las armaduras y el hormigón que las contiene. En éste, como en tantos otros campos de la tecnología, es importante la búsqueda de métodos de ensayo acelerados, que sean a la vez de lo más representativos posible una ayuda para economizar esfuerzo y materiales.

Las técnicas de tipo electroquímico son las que hasta ahora han dado resultados más prometedores en el estudio de la velocidad de corrosión de los metales. En el caso específico del hormigón armado, estas técnicas presentan muchas dificultades a la hora de su aplicación, debido a las especiales características electrolíticas del medio, como son. entre otras: la elevada resistividad específica del hormigón y la no observación directa de los electrodos. Estas dificultades han colaborado a que las técnicas electroquímicas no hayan sido aplicadas en el hormigón tan amplia y exactamente como en otros materiales, y cuando han sido utilizadas, o se han ignorado las limitaciones obteniéndose resultados erróneos, o se han realizado los ensayos sumergiendo directamente las armaduras en disolución saturada de hidróxido cálcico, que si bien presenta unas condiciones termodinámicas (potencial y $\mathrm{pH}$ ) que pueden asimilarse al caso del hormigón endurecido, no se puede hacer lo mismo con las cinéticas, que en ambos medios son diferentes.

Como ya se aludió en un trabajo anterior (1) fueron Kaesche (2) y Baümel y Engell (3) quienes en 1959 comenzaron a aplicar las técnicas electroquímicas a probetas de acero embebidas en mortero, usando el trazado de curvas de polarización potenciocinéticas y galvanostáticas (2) y potenciostáticas (3), en el estudio acelerado del comportamiento de diversos aditivos para hormigón sobre la corrosión de armaduras. A partir de entonces.

(*) C. Andrade.-Dr. en Química Industrial. Colabora en el Dpto. de Química del IETcc y en el el Dpto. de Corrosión y Protección del CENIM.

J. A. González.-Dr. en Química Industrial. Investigador Científico del Dpto. de Corrosión y Protección del CENIM. 
otros autores han vuelto a emplear los citados métodos electroquímicos de carácter acelerado, pero muy pocos de ellos han utilizado ensayos paralelos que constatasen la realidad de las predicciones obtenidas.

En el presente trabajo, continuación de otros anteriores (1) (4), en los que se discutió las ventajas e inconvenientes de los diversos métodos electroquímicos acelerados utilizados hasta el momento (1), así como su correspondencia con ensayos en condiciones naturales de corrosión en disolución saturada de $\mathrm{Ca}(\mathrm{OH})_{2}$ (4), se comunican los resultados obtenidos con redondos embebidos en probetas de mortero endurecido, para condiciones de trabajo similares a las ya utilizadas, y se discuten las limitaciones y posibilidades que como ensayo acelerado presenta la determinación conjunta y paralela de la velocidad instantánea de corrosión (medida de la $R_{\mathrm{p}}$ ) y de los potenciales de ruptura, $E_{\mathrm{r}}$, (métodos galvanostático y potenciodinámico).

\section{MATERIALES Y METODO EXPERIMENTAL}

El mortero se fabricó de acuerdo con el RC-75 (5), con una relación $a / c=0,5$ y cemento/arena de $1 / 3$.

En las probetas que tenían unas dimensiones de $2 \times 5,5 \times 8 \mathrm{~cm}$ se embebieron dos redondos tal y como muestra la fotografía 1, en los que se aislaba la interfase aire-aceromortero con una cinta adhesiva (superficie expuesta al ataque $=11 \mathrm{~cm}^{2}$ ).

Las probetas se curaron las primeras $24 \mathrm{~h}$ a $20^{\circ} \pm 2^{\circ} \mathrm{C}$ y más del $90 \%$ de HR. Luego, unas se introdujeron en vasos de polietilieno, manteniéndose parcialmente sumergidas en agua ( $2 \mathrm{~cm}$ de altura) con el fin de minimizar el efecto perturbador sobre las medidas electroquímicas de la caída óhmica RI, y otras se conservaron a la atmósfera del laboratorio, $\left(20^{\circ} \pm 2^{\circ} \mathrm{C}\right.$ y $40 \%$ de HR $)$.

Los aceros fueron del mismo tipo (para pretensado) que los utilizados en (1) y (4) y se prepararon de igual forma que la allí descrita. Se ensayaron un total de 56 redondos (28 probetas).

El cemento utilizado fue un P-350 de análisis químico: P.F. $=3,7 \%$; RI $=2,7 \%$; $\mathrm{SiO}_{2}=18,1 \% ; \mathrm{Al}_{2} \mathrm{O}_{3}=7,0 \% ; \mathrm{Fe}_{2} \mathrm{O}_{3}=$ $=3,0 \% ; \mathrm{CaO}=60,2 \% ; \mathrm{MgO}=2,1 \%$; $\mathrm{SO}_{3}=3,2 \% ; \mathrm{CaO}$ libre $=1,3 \%$ y superficie específica $=3.338 \mathrm{~cm}^{2} / \mathrm{g}$.

Como aditivos se utilizaron, en diversas proporciones (siempre en relación al peso de cemento) y con la pureza exigida en los reac-

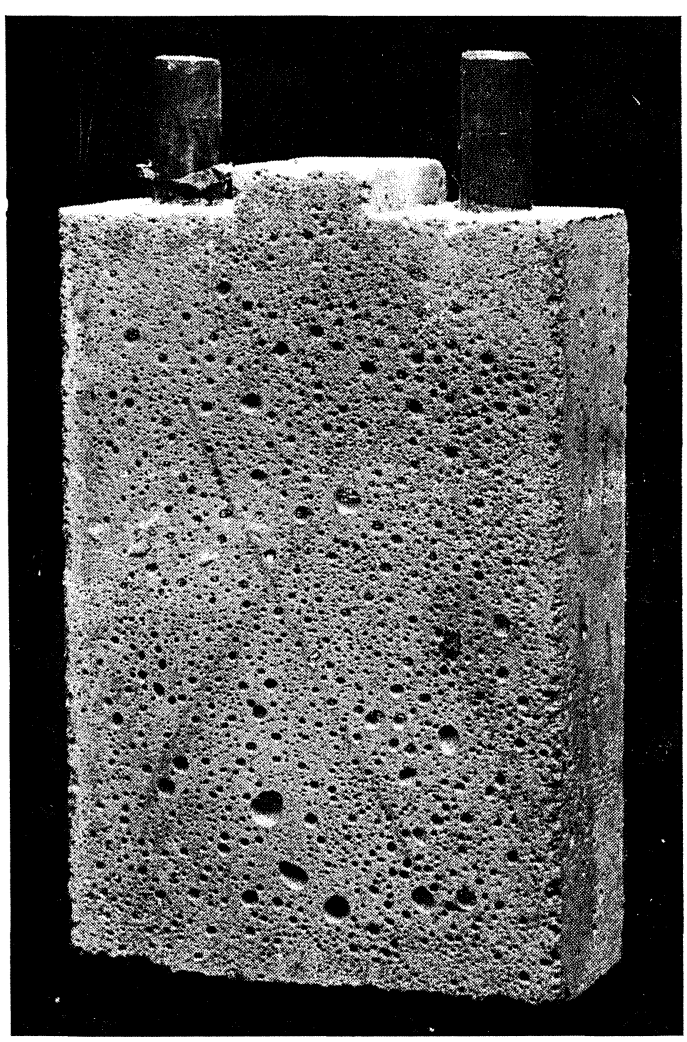

Fotografía n.o 1 tivos para análisis: $\mathrm{Cl}_{2} \mathrm{Ca}$ y $(\mathrm{HCOO})_{2} \mathrm{Ca}$ (que se utiliza en ocasiones como acelerante de fraguado en sustitución del cloruro cálcico) como presuntos despasivantes, y $\mathrm{NO}_{2} \mathrm{Na}$ como inhibidor de corrosión. 
Las medidas electroquímicas (toma de $R_{\mathrm{p}}$ y trazado de la rama anódica de las curvas de polarización) se llevaron a cabo de igual forma que la descrita en (4), con un potenciostato de la casa AMEL, modelo Metalloscan.

La $I_{\text {corr }}$ se calculó a partir de la determinación de $R_{\mathrm{p}}$, mediante la fórmula de Stern: $I_{\text {corr }}=B / R_{\mathrm{p}}$ eligiendo como valores de la constante $B$ el de $0,026 \mathrm{~V}$ para el acero en estardo activo y el de $0,052 \mathrm{~V}$ para el mismo material en estado pasivo (6). El potencial de corrosión, $E_{\text {corr }}$, se midió frente al electrodo de calomelanos saturado (ES..S.).

\section{RESULTADOS}

En las figuras 1 a 4 se representan la $I_{\text {corr }} \mathrm{y}$ los $E_{\text {corr }}$ relativos a los redondos ensayados bajo diferentes condiciones de agresividad, según fuesen empleados o no aditivos, la naturaleza de éstos y el tipo de exposición de las probetas (parcialmente sumergidas o en la atmósfera).

En la fig. 1 se muestra el comportamiento de tres redondos embebidos en mortero que: (A) no contenía aditivo, $(B)$ contenía $3 \%$ de formiato cálcico y $(C)$ un $2 \% \mathrm{Cl}_{2} \mathrm{Ca}+$ $+4 \% \mathrm{NO}_{2} \mathrm{Na}$. Todas estas probetas de mortero se mantuvieron parcialmente sumergidas durante los ensayos y puede deducirse tanto de los valores de $I_{\text {corr }}$, como de $E_{\text {corr }}$, que el acero no sufrió ataque apreciable, manteniéndose pasivo, hecho que se comprobó posteriormente al romper las probetas al final del ensayo. Es interesante destacar, dentro de su pequeño valor total, el mayor ataque exhibido durante la primera semana por los redondos embebidos en mortero con formiato cálcico.

En la figura 2 se reproducen los resultados para cuatro redondos embebidos en mortero con $2 \%$ de $\mathrm{Cl}_{2} \mathrm{Ca}$, conservados en cámara húmeda ( $\left.\mathrm{HR}>90 \%\right)$ uno, $(E)$, y parcialmente sumergidos los otros tres: $(F),(G)$ y $(H)$. Se quiere llamar la atención sobre los hechos siguientes: 1) que los redondos $(F)$ y $(G)$, que estaban embebidos ambos en la misma probeta, presentan velocidades de corrosión completamente diferentes, y 2) que los $E_{\text {corr }}$ del redondo $(H)$, a partir de las dos semanas de vida, aproximadamente, se hacen cada vez más negativos, no evolucionando paralelamente la $I_{\text {corr }}$. Después de romper las probetas, se pudo comprobar que el $(E)$ y el $(F)$ habían sufrido bastante ataque, con picaduras distribuidas por toda su superficie, mientras que $(G)$ presentaba sólo dos pequeñísimas picaduras y $(H)$ tenía ligeramente corroída toda la línea de contacto con la cinta aislante.

En la figura 3 se muestra la influencia del contenido en humedad del mortero para el caso de tres redondos: (I) embebido en mortero con $1 \%$ de $\mathrm{Cl}_{2} \mathrm{Ca},(J)$ con $2 \% \mathrm{de}^{\mathrm{Cl}} \mathrm{Cl}_{2} \mathrm{Ca}$ y $(K)$ con $2 \% \mathrm{Cl}_{2} \mathrm{Ca}+2 \% \mathrm{NO}_{2} \mathrm{Na}$. Se conservaron durante 30 días en la atmósfera del laboratorio: en los primeros días, el $(J)\left(2 \% \mathrm{Cl}_{2} \mathrm{Ca}\right)$ sufre una fuerte corrosión, registrando $I_{\text {corr }}$ bastante elevadas, que son menores para el $(I)\left(1 \% \mathrm{Cl}_{2} \mathrm{Ca}\right)$ e insignificantes para el $(K)\left(2 \% \mathrm{Cl}_{2} \mathrm{Ca}+2 \% \mathrm{NO}_{2} \mathrm{Na}\right)$ que parece estar pasivado desde el principio. Después de la primera semana todos presentan unas velocidades de corrosión muy pequeñas, y unos $E_{\text {corr }}$ más nobles que $-300 \mathrm{mV}$, indicando que todos los redondos han alcanzado el estado pasivo. A los 30 días se humedecieron las probetas sumergiéndolas parcialmente en agua, produciéndose inmediatamente un salto de los $E_{\text {corr }}$ hacia valores muy negativos y un aumento importante en la $I_{\text {corr }}$, menos acusado para el redondo $(K)$ que contenía $\mathrm{NO}_{2} \mathrm{Na}$ como inhibidor. Después de romper las probetas pudo comprobarse efectivamente que el redondo $(K)$ presentaba mucha menos corrosión que los (I) y $(J)$, los cuales mostraban picaduras por varias zonas de su superficie. 


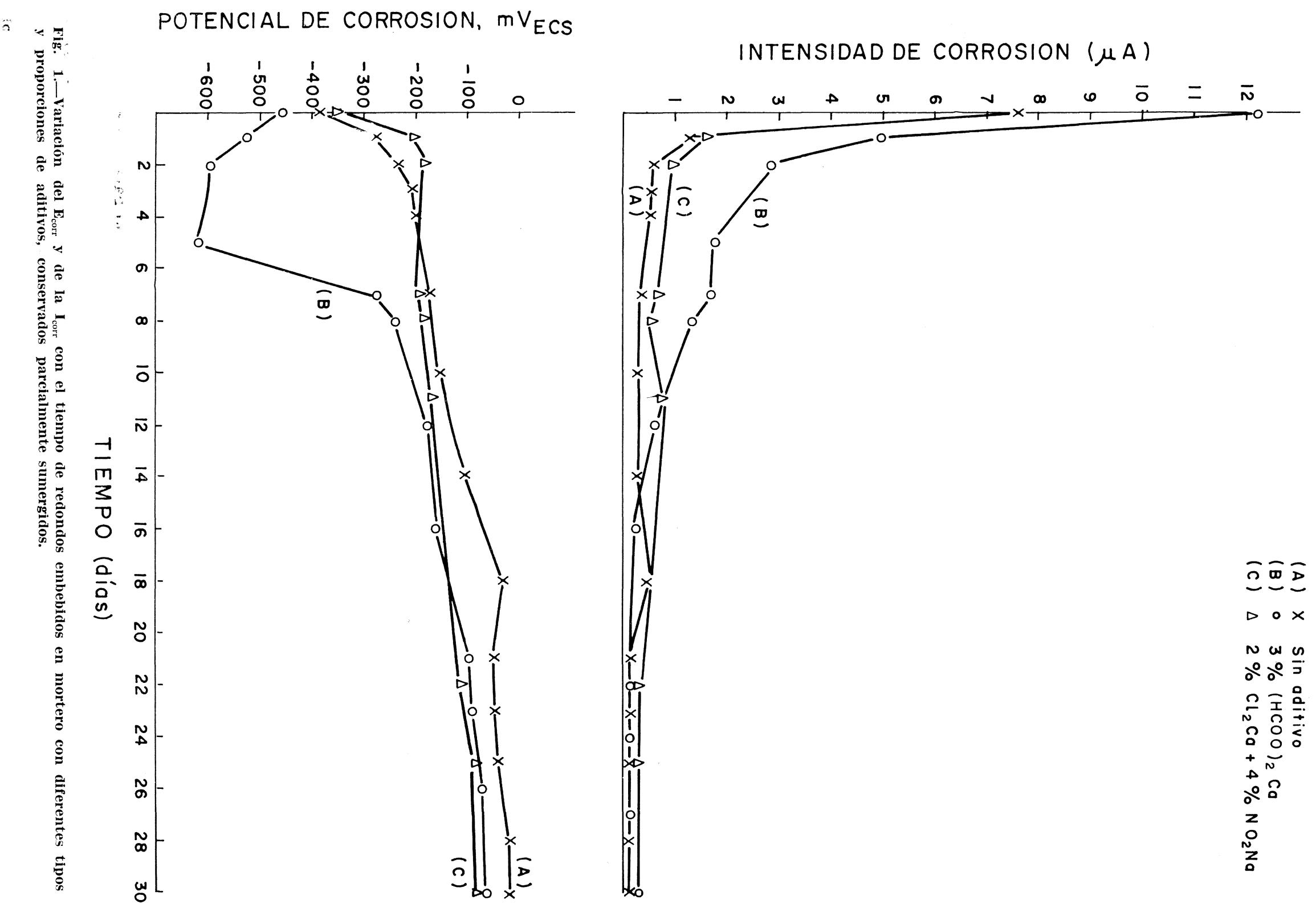



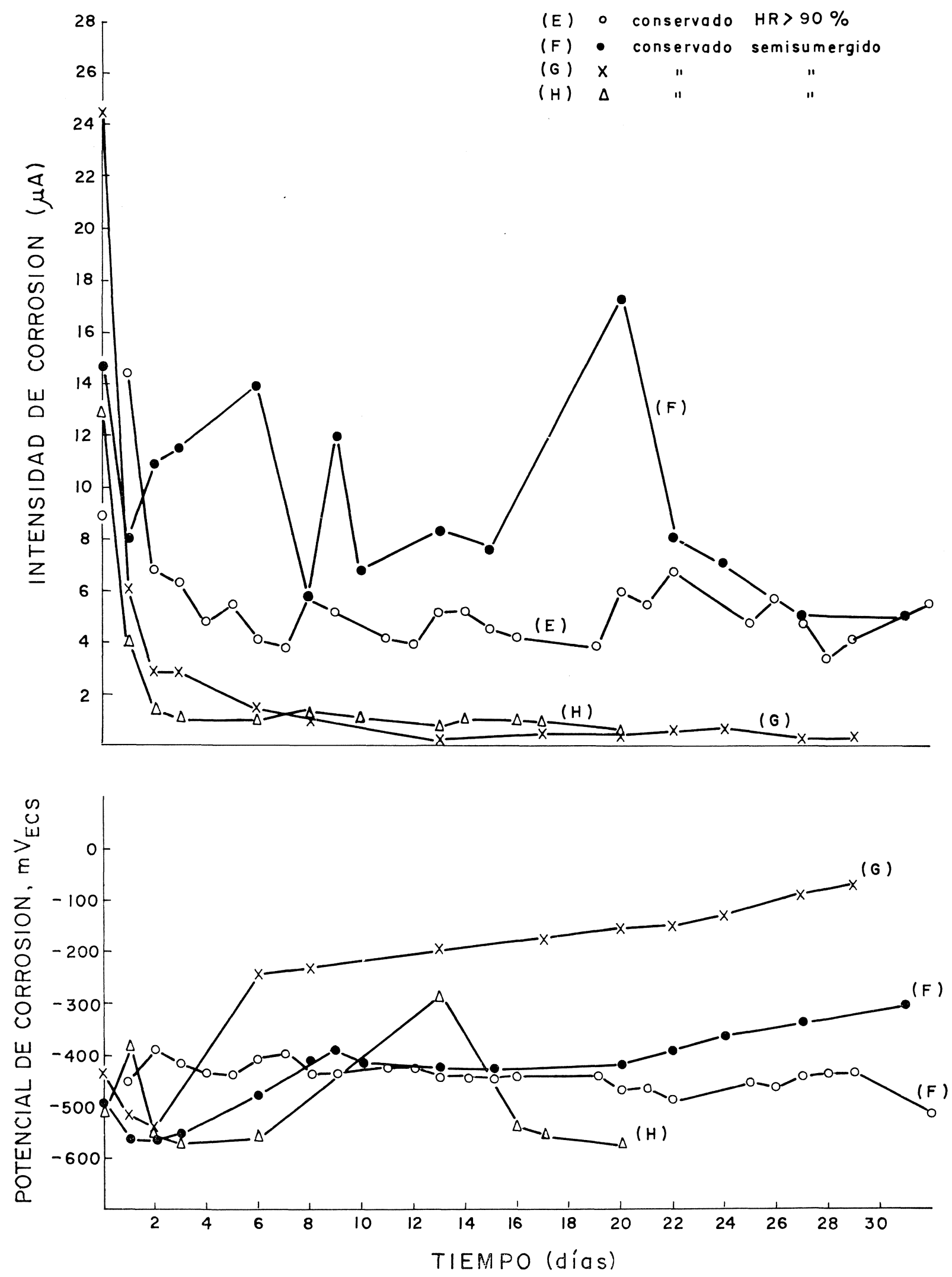

Fig. 2.-Variación del $\mathbf{E}_{\text {corr }} \mathbf{y}$ de $1 a \mathrm{I}_{\text {corr }}$ con el tiempo de redondos embebidos en mortero con $2 \%$ de $\mathrm{Cl}_{2} \mathrm{Ca}$ y conservados en ambientes con distinto contenido en humedad. 

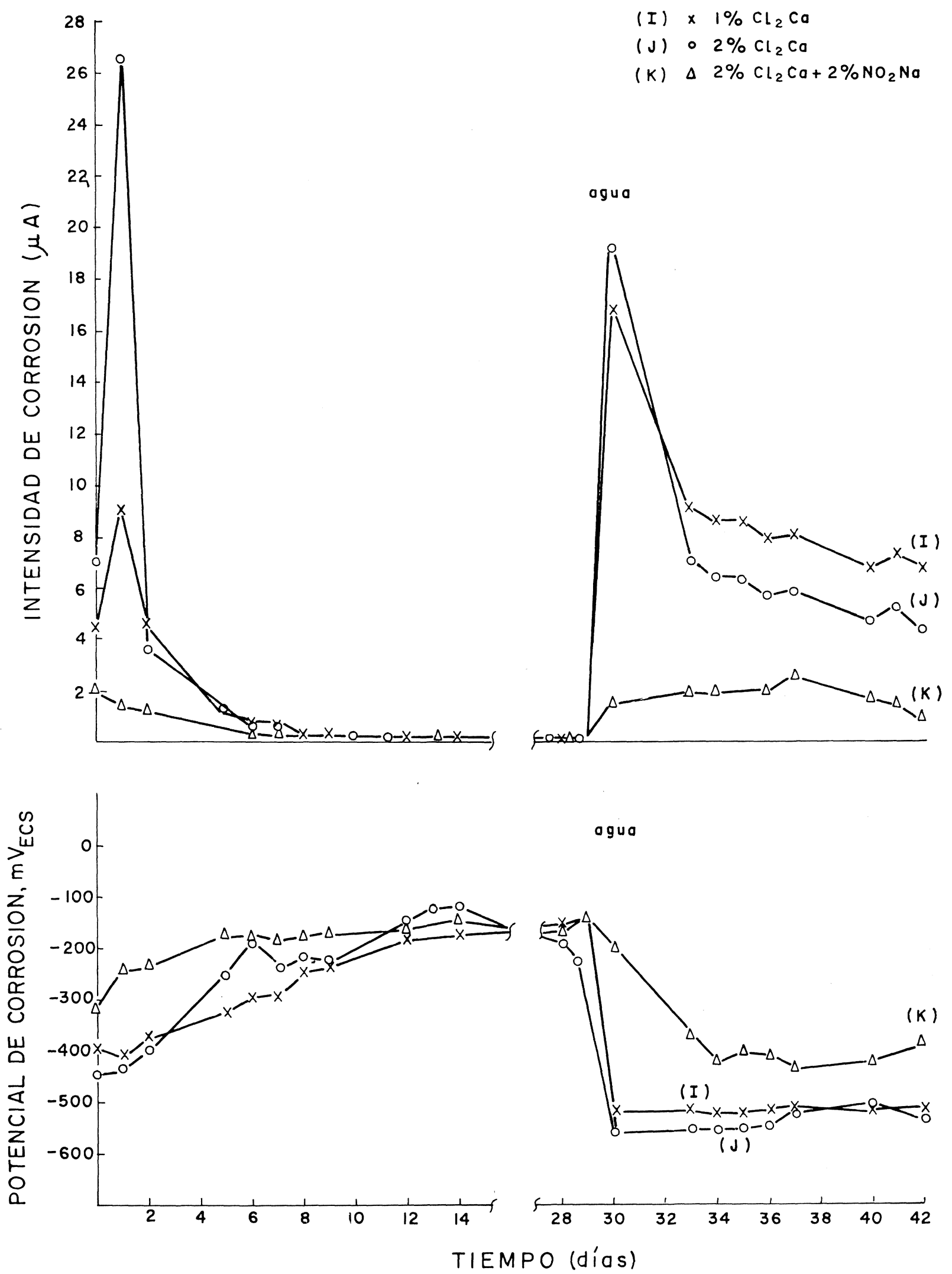

TIEMPO (días)

Fig. 3.-Variación del $E_{\text {corr }} y$ de la $I_{\text {corr }}$ con el tiempo de redondos embebidos en mortero con diferentes tipos $y$ proporciones de adtivos conservados primero en ambiente seco y parcialmente sumergidos a partir de los 30 días. 
Finalmente, en la figura 4, se muestra en coordenadas doble logarítmicas la correspondencia que aparece entre las pérdidas de peso obtenidas gravimétricạmente y las calculadas a partir de los valores de $R_{\mathrm{p}}$, de aquellos redondos en los que se efectuaron ambas determinaciones. Los valores gravimétricos son casi siempre mayores que los electroquímicos, circunstancia que se justifica por el hecho de que los aparatos de medida empleados con los redondos, representados en la figura, no eliminan automáticamente la caída óhmica RI a través del hormigón, deduciéndose electroquímicamente unas $I_{\text {corr }}$ menores (entre el $20 \%$ y el $40 \%$ ) que las reales. Una explicación más amplia de esta circunstancia se ha realizado en (6).

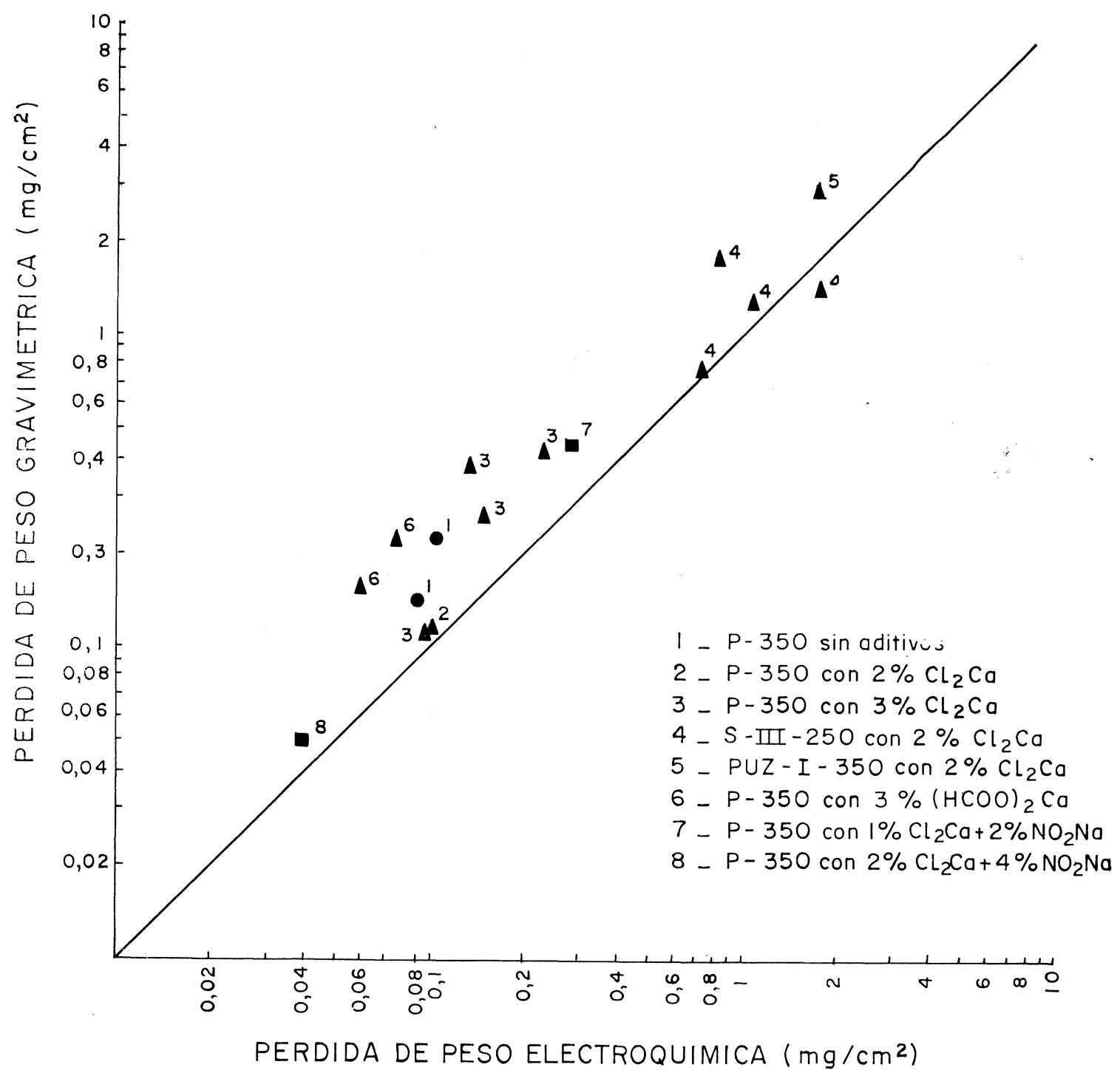

Fig. 4.-Pérdidas de peso gravimétricas y electroquímicas de redondos enbebidos en mortero con diferentes tipos y proporciones de adtivos.

En las figuras 5 a 8 se representa la evolución del potencial al aplicar galvanostáticamente $10 \mu \mathrm{A} / \mathrm{cm}^{2}$ a los redondos embebidos en las probetas de mortero, tanto a las parcialmente sumergidas como a las conservadas en la atmósfera del laboratorio.

Se utilizó un acero nuevo para cada curva realizada (1), y al finalizar los ensayos se procedió en todos los casos a la rotura de las probetas para observación visual de los redondos. Se comprobó que en todos los casos que aparece un potencial de ruptura en las cur- 
vas, existían picaduras en los redondos, mientras que éstos aparecían completamente limpios de óxido en aquellos otros casos en que las curvas no detectaban rotura de la capa pasivante.

En la figura 5 se muestran las curvas de polarización galvanostática, tomadas recién desenmoldadas las probetas, a las $24 \mathrm{~h}$ de su fabricación. Las curvas correspondientes a las probetas que no contenían aditivos o contenían un $3 \%$ de formiato cálcico, presentan un trazado similar, sin aparición de $E_{\mathrm{r}}$, de lo que se deduce que no hay riesgo de ataque. Con el resto de los aditivos aparecen unos $E_{\mathrm{r}}$, tanto más nobles cuanto menor es el contenido en $\mathrm{Cl}_{2} \mathrm{Ca}$.

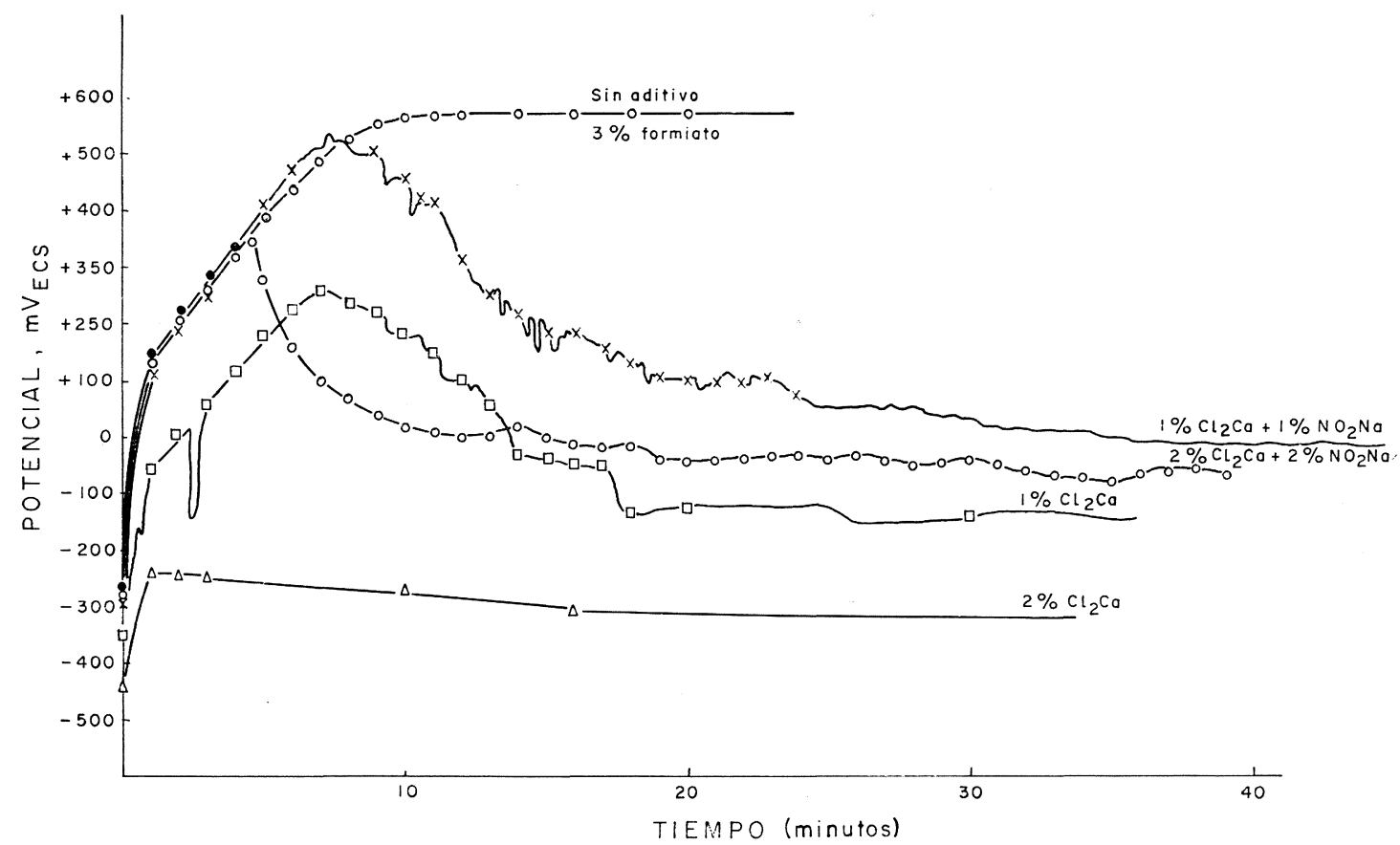

Fig. 5.-Curvas galvanostáticas $\left(10 \mu \mathrm{A} / \mathrm{cm}^{2}\right)$ de redondos embebidos en mortero con diferentes tipos y proporciones de aditivos a las $24 \mathrm{~h}$ de su fabricación.

La figura 6 muestra el mismo tipo de curvas realizadas en probetas conservadas durante 3 días en ambiente seco. Mientras que en el caso del $1 \%$ y $2 \%$ de $\mathrm{Cl}_{2} \mathrm{Ca}$ y $2 \% \mathrm{Cl}_{2} \mathrm{Ca}+$ $+2 \% \mathrm{NO}_{2} \mathrm{Na}$ siguen apareciendo $E_{\mathrm{r}}$, indicando un riesgo de corrosión localizada (menor cuanto más alejado está el $E_{\mathrm{r}}$ de $E_{\text {corr }}$ ); en las probetas que no contienen aditivo o contienen $3 \%$ de formiato cálcico o $1 \% \mathrm{Cl}_{2} \mathrm{Ca}+1 \% \mathrm{NO}_{2} \mathrm{Na}$, el riesgo de formación de picaduras no existe al no aparecer ningún $E_{\mathrm{r}}$.

La figura 7 reproduce las curvas para las mismas condiciones que la figura anterior (ambiente seco) pero a los 7 días de vida de las probetas. Se puede observar que en este caso todas las curvas alcanzan el valor del potencial de desprendimiento de $\mathrm{O}_{2}$ y mientras con el $2 \%$ de $\mathrm{Cl}_{2} \mathrm{Ca}$ se produce inmediatamente la rotura de la capa pasivante, con el $1 \%$ son necesarios más de 20 minutos. En el resto de los casos ha desaparecido el riesgo de corrosión localizada por estar suficientemente fortalecida la capa pasivante. 


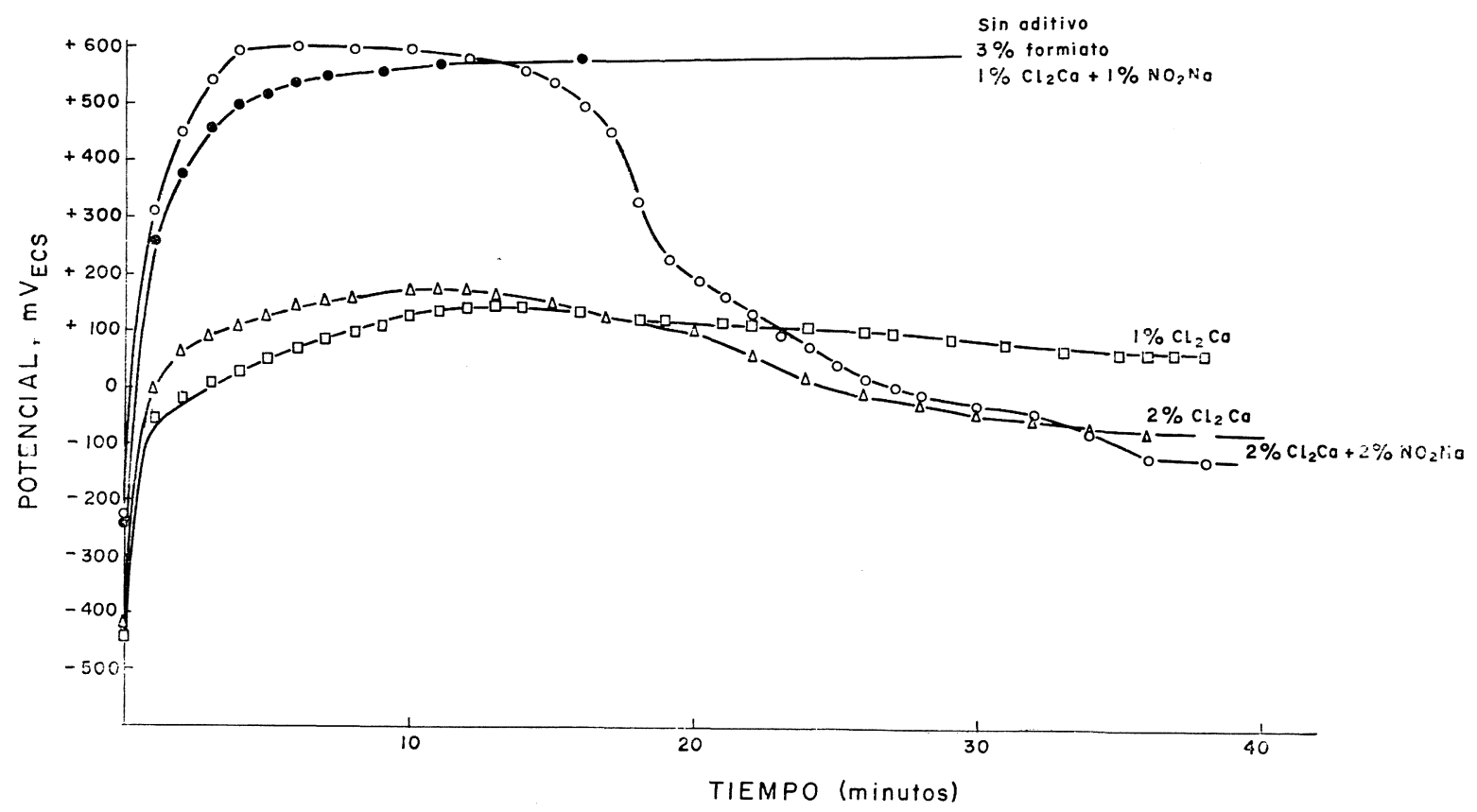

Fig. 6. -Curvas galvanostáticas $\left(10 \mu \mathrm{A} / \mathrm{cm}^{2}\right)$ de redondos embebidos en mortero con diferentes tipos y proporciones de aditivos conservados en atmósfera seca, a los 3 días de su fabricación.

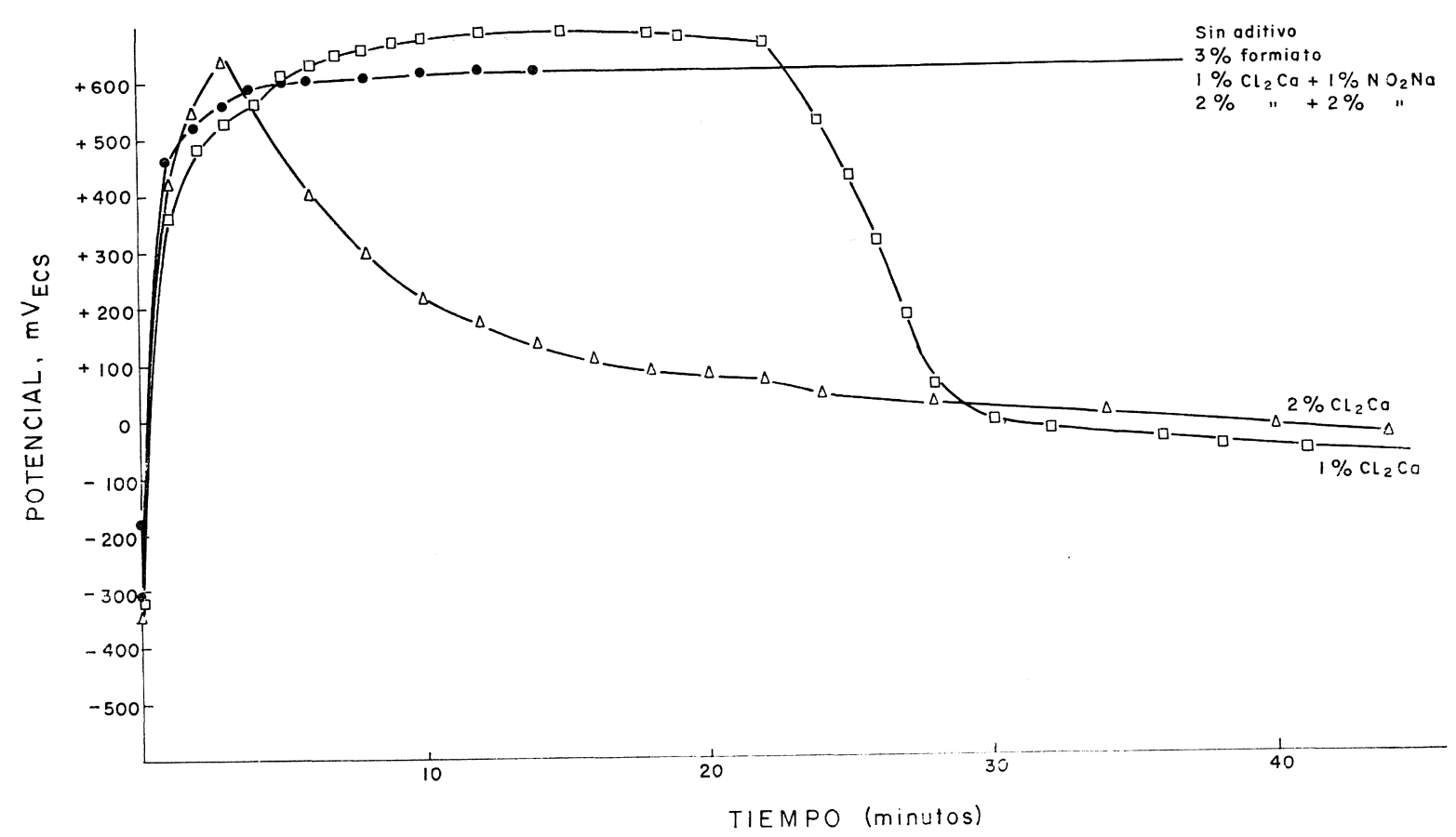

Fig. 7.-Curvas galvanostáticas $\left(10 \mu \mathrm{A} / \mathrm{cm}^{2}\right)$ de redondos embebidos en mortero con diferentes tipos y proporciones de aditivos conservados en atmósfera seca, a los 7 días de su fabricación.

En la figura 8 aparecen las curvas galvanostáticas realizadas sobre redondos embebidos en mortero que se conservó parcialmente sumergido durante 7 días. Puede apreciarse que en estas condiciones aumenta notablemente el riesgo de deterioro de la capa pasivante, pues excepto en las probetas sin aditivo y en las que contienen $3 \%$ de formiato cálcico, en todas las demás aparecen $E_{\mathrm{r}}$ (muy cercano al correspondiente $E_{\text {corr }}$ en el caso del $2 \%$ de $\mathrm{Cl}_{2} \mathrm{Ca}$ ), y con un tiempo de incubación de picaduras que aumenta (disminuye el riesgo de corrosión) con la disminución en el contenido de $\mathrm{Cl}_{2} \mathrm{Ca}$. 


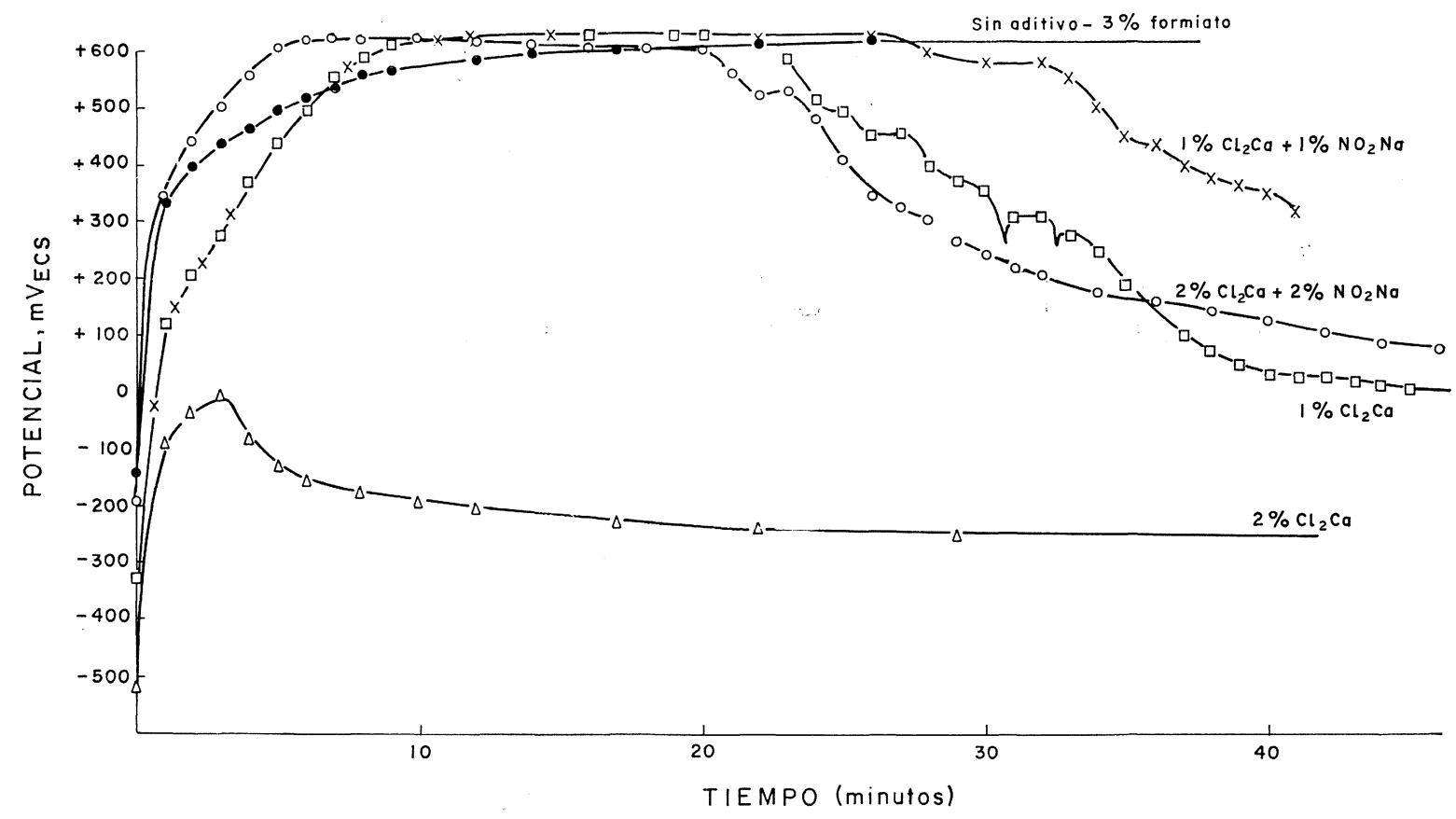

Fig. 8.-Curvas galvanostáticas $\left(10 \mu \mathrm{A} / \mathrm{cm}^{2}\right)$ de redondos embebidos en mortero con diferentes tipos $y$ proporciones de adtivos conservados parcalmente sumergidos, a los $\gamma$ días de su fabricación.

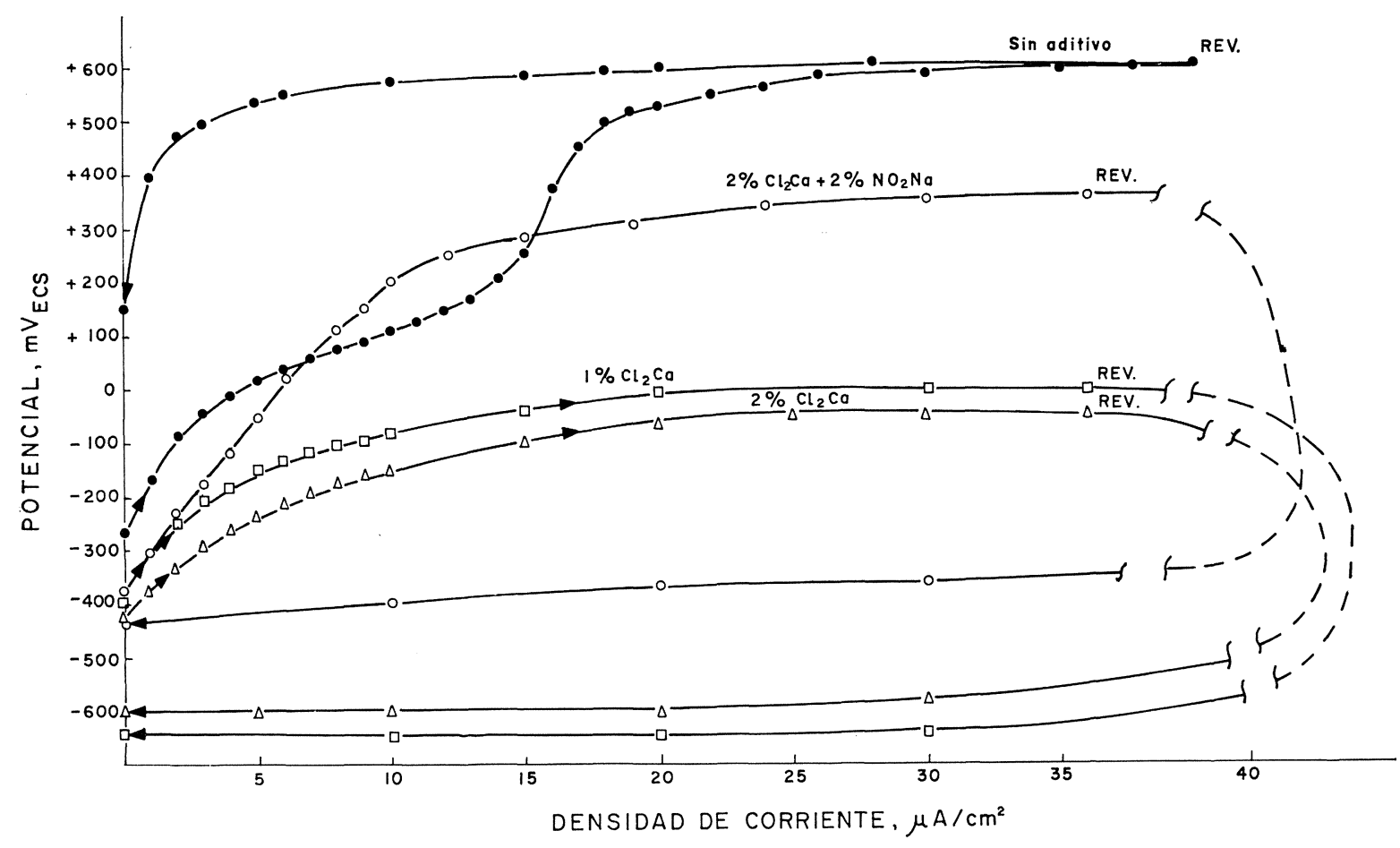

Fig. 9.-Curvas potenciodinámicas $(50 \mathrm{mV} / \mathrm{min})$ de redondos embebidos en mortero con diferentes tipos $y$ proporciones de aditivos a las $24 \mathrm{~h}$ de su fabricación.

Finalmente en la figura 9 se muestran las curvas potenciodinámicas, tanto de potenciales crecientes como decrecientes (1), para alguno de los casos considerados anteriormente: de ellas se extraen deducciones muy similares a todas las procedentes, en relación con los valores relativos de los distintos $E_{\mathrm{r}}$. No se realizaron más curvas potenciodinámicas en este caso de probetas de mortero, por considerar que llegando a conclusiones semejantes con las galvanostáticas, son más ilustrativas éstas. 


\section{DISCUSION}

\subsection{Comparación con los ensayos en disolución saturada de $\mathrm{Ca}(\mathrm{OH})_{2}$}

Las diferencias encontradas entre los ensayos realizados en disolución saturada de $\mathrm{Ca}(\mathrm{OH})_{2}$ y los realizados en mortero endurecido hay que atribuirlas al impedimento físico que supone la capa de hormigón para la difusión del oxígeno, humedad y otras sustancias agresivas para las armaduras.

La cinética del proceso es cuantitativamente diferente en ambos medios, siendo las $I_{\text {corr }}$ presentadas por las probetas en disolución más elevadas que las correspondientes a las embebidas en mortero, pero cualitativamente el comportamiento de ambas es bastante similar. Cuando las armaduras resultan protegidas, después de las primeras $24 \mathrm{~h}$ durante las que las $I_{\text {corr }}$ pueden tomar valores incluso elevados, debido a que todavía no se ha consolidado suficientemente la capa pasivante, la velocidad de disolución del acero se hace cada vez más inapreciable. Si falla tal protección, bien sea porque no se forme la capa pasivante o por rotura de la misma, entonces se registran unas $I_{\text {corr }}$ elevadas, frecuentemente con un máximo que suele situarse en las primeras $48 \mathrm{~h}$, aunque no en los primeros momentos, para alcanzar luego un valor más o menos constante, elevado si continúa el ataque, e inapreciable si se pasivan las picaduras formadas.

Finalmente, lo mismo que sucedía en disolución saturada de $\mathrm{Ca}(\mathrm{OH})_{2}$, la cinética del ataque parece llegar a una estabilización mucho antes de que se alcancen los 30 días de vida a los que se han extendido la mayoría de los ensayos de forma que si no cambian las condiciones ambientales del sistema medio-hormigón armado, se pueden realizar predicciones del comportamiento futuro a partir de tales resultados.

\subsection{Significado del potencial de corrosión}

Ya en trabajos anteriores (1) (4) se ha destacado el carácter termodinámico del $E_{\text {corr }}$ (indica un estado o situación del metal: pasividad, inmunidad, etc.), y las limitaciones de esta variable como índice de la cinética de corrosión (no cuantifica la proporción de metal por unidad de área que se corroe). Los datos del presente trabajo aportan nueva confirmación de esta idea. Así, la curva $(H)$ de la figura 2 muestra que a un descenso del $E_{\text {corr }}$ no le corresponde en todos los casos un aumento simultáneo de la $I_{\text {corr }}$, como también se ha observado que para un mismo $E_{\text {corr }}$ se estiman (según el tipo de cemento, aditivo y medio de conservación) valores de $I_{\text {corr }}$ que en algunos casos son hasta 5-6 veces más elevados que en otros.

Todo ello pone de relieve que, aunque sería muy sugestivo poder deducir velocidades de corrosión a partir de medidas tan simples como la del potencial de corrosión, sus indicaciones en cuanto a la cinética del ataque, no deben considerarse más que como orientativas y de validez estrictamente cualitativa, sin que se pueda establecer de una forma general y para todas las condiciones de exposición una relación biunívoca cuantitativa entre el $E_{\text {corr }}$ y la cinética del proceso.

\subsection{Aditivos empleados}

A la vista de la consideración conjunta de los valores de los potenciales de ruptura y de la $R_{\mathrm{p}}$, los aditivos que se añaden al hormigón para mejorar o modificar alguna de sus cualidades, podrían clasificarse respecto de la corrosión de armaduras, en una primera aproximación, en "potencialmente corrosivos", "inocuos" e "inhibidores". 
Se ha preferido nombrar a los aditivos con iones desapasivantes como "potencialmente" corrosivos puesto que, si su presencia es necesaria para que se produzca la rotura de la capa pasivante, necesitan la concurrencia de otras circunstancias, como pueden ser la presencia simultánea de un mínimo de humedad y acceso de oxígeno, o la creación de diferencias de potencial que anulen la existente entre $E_{\text {corr }}$ y $E_{\mathrm{r}}$, bien sea por pilas de aireación diferencial, $\mathrm{pH}$ diferencial (carbonatación), etc. Recuérdese a este respecto que las pilas de $\mathrm{pH}$ diferencial pueden generar diferencias de potencial del orden de $0,5 \mathrm{~V}$ (7). La existencia de un $E_{\mathrm{r}}$ en las curvas galvanostáticas no significa pues que las armaduras se vayan necesariamente a corroer, sino que indica un "peligro potencial de corrosión".

Tal es el caso del 1,2 y $3 \%$ de $\mathrm{Cl}_{2} \mathrm{Ca}$ [curvas $(H)$ y $(G)$ de la figura 2 e $(I)$ y $(J)$ de la figura 3], que muestran ejemplos en que la presencia de potenciales de ruptura no se traduce necesariamente en una elevada $I_{\text {corr }}$ estimada a partir de las medidas de $R_{\mathrm{p}}$ y, asimismo, los redondos $(F)$ y $(G)$ embebidos en la misma probeta de mortero que evidencian conductas muy diferentes. Una aproximación a la comprensión de estos comportamientos erráticos podría llevar a deducir que, en el primer caso [curvas $(H),(G)$, $(I),(J)]$, la cantidad de iones $\mathrm{Cl}^{-}$libres en la fase líquida es muy pequeña, debido a que la mayoría del $\mathrm{Cl}_{2} \mathrm{Ca}$ añadido se ha combinado con los aluminatos del cemento P-350 normal utilizado, para dar cloroaluminatos, que son compuestos no agresivos; y en el segundo caso [curvas $(F)$ y $(G)$ ] un desigual reparto de los citados iones $\mathrm{Cl}^{-}$libres o un diferente estado superficial del acero a escala microscópica, inducen la formación de pilas más activas en un redondo que en otro.

Algo parecido (reacción con las fases del cemento para dar compuestos hidratados no agresivos), parece ocurrir con los formiatos, que en disolución saturada de $\mathrm{Ca}(\mathrm{OH})_{2}$ se ha comprobado que actúan como despasivantes (1), mientras que en mortero, aún utilizados en cantidades superiores, se han mostrado como inocuos.

En cuanto a los nitritos, utilizados como inhibidores de los cloruros, muestran un comportamiento similar al observado en los ensayos en disolución. Las medidas de $R_{\mathrm{p}}$ prueban que el ataque de las armaduras es despreciable siempre que la dosis de $\mathrm{NO}_{2} \mathrm{Na}$ sea al menos igual a la de $\mathrm{Cl}_{2} \mathrm{Ca}$; ahora bien, las curvas galvanostáticas trazadas en los primeros días muestran, sobre todo con mortero húmedo, unos potenciales de ruptura que indican un riesgo de corrosión cuando por circunstancias intrínsecas (por ejemplo aumento de humedad) o extrínsecas (por ejemplo, corrientes vagabundas) al medio, el $E_{\text {corr }}$ se desplazase durante suficiente tiempo hacia valores más anódicos que el correspondiente $E_{\mathrm{r}}$ o viceversa. En estos casos, el riesgo desaparece cuando pasa el tiempo suficiente para que los nitritos hayan consolidado la capa pasivante. Como ya se ha mencionado en otros trabajos (1), entre los autores que han utilizado el nitrito sódico como inhibidor de la corrosión de las armaduras, no hay acuerdo total en cuanto a la efectividad y duración de su carácter protector. Realmente, si la humedad del mortero es suficiente, existe riesgo de ataque (que disminuye hasta desaparecer, con el paso del tiempo), a no ser que se utilicen relaciones $\mathrm{NO}_{2}{ }^{-} / \mathrm{Cl}^{-}$elevadas.

En la tabla 1 se intenta resumir el efecto sobre los potenciales de ruptura, del tiempo, condiciones de exposición y cuantía y naturaleza de los aditivos empleados. Se ve, que con el transcurso del tiempo, aumenta la diferencia entre $E_{\mathrm{r}}$ y $E_{\text {corr }}$, disminuyendo por lo tanto el riesgo de que cualquier pila de corrosión desencadene el ataque por picaduras; que los formiatos no parecen crear susceptibilidad al ataque localizado bajo ninguna circunstancia; que los nitritos atenúan el carácter desfavorable de los cloruros, eliminándolo cuando el ambiente es seco o se añaden en concentración suficiente y, finalmente, que la inmersión parcial es una situación mucho más agresiva que la exposición a una atmósfera relativamente seca. Todas estas características habían sido ya puestas de manifiesto por estimaciones de la velocidad de corrosión a partir de las medidas de $R_{\mathrm{p}}$. 
Finalmente, se quiere recordar aquí que el valor absoluto del potencial de ruptura, $E_{\mathrm{r}}$. determinado galvanostáticamente, es función no sólo de la naturaleza del metal y del medio, sino también de la densidad de corriente aplicada, $10 \mu \mathrm{A} / \mathrm{cm}^{2}$ en este caso, por lo que los valores no deben tomarse más que con validez comparativa para condiciones totalmente similares. Si se quiere llegar a unos valores realmente fiables de $E_{\mathrm{r}}$ es necesario recurrir al método potenciostático, mucho más laborioso (4).

\subsection{Contenido en humedad}

Es de destacar la importancia de la humedad como factor determinante de la intensidad del ataque, de tal forma que en un mortero seco puede detenerse casi por completo la corrosión iniciada por la presencia de cloruros y, si se humedece la probeta, volver a alcanzar valores notables, según prueba la figura 3 .

Se ha comprobado al respecto, aunque los resultados no se exponen en este trabajo, que generalmente la sîuación más desfavorable no es aquella en la que el mortero se halla saturado de humedad, por ejemplo cuando está sumergido totalmente, pues entonces sus poros se llenan de agua y el oxígeno necesario para la semirreacción catódica sólo puede llegar por la previa disolución y difusión en la fase acuosa que ocupa los poros, sino aquella en que el mortero se encuentra en ambientes muy húmedos, HR $>90 \%$, circunstancia en la que, aparte de existir suficiente humedad para el progreso de la semirreacción anódica, el oxígeno necesario para la catódica alcanza con menor dificultad la superficie de las armaduras.

\section{TABLA 1}

Evolución de los potenciales de ruptura de las armaduras embebidas en mortero, en función del tiempo, las condiciones de exposición, y la cuantía y naturaleza de los aditivos empleados

\begin{tabular}{|c|c|c|c|c|c|c|c|}
\hline \multirow{2}{*}{$\begin{array}{l}\text { Condiciones de } \\
\text { exposición }\end{array}$} & \multirow{2}{*}{ Aditivos empleados } & \multicolumn{3}{|c|}{ Potenciales de ruptura $(\mathrm{mV})$} & \multicolumn{3}{|c|}{$\begin{array}{l}\text { Diferencia entre } \\
E_{\mathrm{r}}-E_{\text {corr }}\end{array}$} \\
\hline & & $24 \mathrm{~h}$ & 3 días & I días & $24 \mathrm{~h}$ & 3 días & z días \\
\hline $\begin{array}{l}\text { Exposición a } \\
\text { la atmósfera }\end{array}$ & \begin{tabular}{ll} 
& \multicolumn{2}{c}{ Sin aditivos } \\
$3 \%$ & $(\mathrm{HCOO})_{2} \mathrm{Ca}$ \\
1 & $\% \mathrm{Cl}_{2} \mathrm{Ca}+1 \% \mathrm{NO}_{2} \mathrm{Na}$ \\
2 & $\% \mathrm{Cl}_{2} \mathrm{Ca}+2 \% \mathrm{NO}_{2} \mathrm{Na}$ \\
1 & $\% \mathrm{Cl}_{2} \mathrm{Ca}$ \\
2 & $\% \mathrm{Cl}_{2} \mathrm{Ca}$
\end{tabular} & $\begin{array}{l}\text { no hay } E_{\mathrm{r}} \\
\text { no hay } E_{\mathrm{r}} \\
+530 \\
+350 \\
+260 \\
-360\end{array}$ & $\begin{array}{l}\text { no hay } E_{\mathrm{r}} \\
\text { no hay } E_{\mathrm{r}} \\
\text { no hay } E_{\mathrm{r}} \\
\quad+600 \\
+150 \\
+180\end{array}$ & $\begin{array}{l}\text { no hay } E_{\mathrm{r}} \\
\text { no hay } E_{\mathrm{r}} \\
\text { no hay } E_{\mathrm{r}} \\
\text { no hay } E_{\mathrm{r}} \\
\quad+690 \\
+650\end{array}$ & $\begin{array}{l}- \\
- \\
800 \\
630 \\
610 \\
210\end{array}$ & $\begin{array}{l}- \\
- \\
- \\
820 \\
600 \\
600\end{array}$ & $\begin{array}{l}- \\
- \\
- \\
- \\
1.000 \\
980\end{array}$ \\
\hline $\begin{array}{c}\text { Probetas } \\
\text { parcialmente } \\
\text { sumergidas }\end{array}$ & 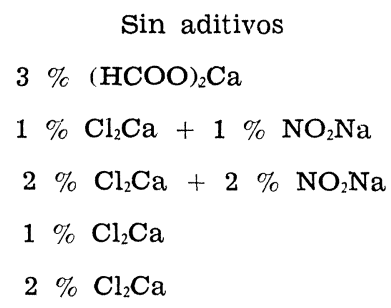 & $\begin{array}{l}- \\
- \\
- \\
- \\
- \\
-\end{array}$ & $\begin{array}{l}- \\
- \\
- \\
-\end{array}$ & $\begin{array}{l}\text { no hay } E_{\mathrm{r}} \\
\text { no hay } E_{\mathrm{r}} \\
+620 \\
+620 \\
+600 \\
-\quad 10\end{array}$ & $\begin{array}{l}- \\
- \\
- \\
- \\
-\end{array}$ & $\begin{array}{l}- \\
- \\
- \\
- \\
-\end{array}$ & $\begin{array}{l}- \\
- \\
950 \\
820 \\
800 \\
520\end{array}$ \\
\hline
\end{tabular}




\section{EMPLEO CONJUNTO DE LA $R_{\mathrm{p}}$ Y LOS $\mathrm{E}_{\mathrm{r}}$ PARA DETERMINAR EL EFECTO DE LOS FACTORES INDIVIDUALES DE CORROSION SOBRE EL ATAQUE DE LAS ARMADURAS}

Como ya se aludió en el apartado 1., el trazado de curvas de polarización, a nivel de laboratorio especializado, se ha venido utilizando desde el año 1959 para determinar, de forma acelerada, la capacidad corrosiva de determinados aditivos, en especial el $\mathrm{Cl}_{2} \mathrm{Ca}$, presentando las limitaciones inherentes a todo ensayo acelerado, sobre todo en lo referente a la no reproducción fidedigna de las condiciones reales de trabajo.

A.simismo, se ha utilizado la determinación de la velocidad de corrosión, sea mediante medidas gravimétricas, como electroquímicas, para conocer el desarrollo e influencia en el ataque de unas determinadas circunstancias, presentando, (las electroquímicas), a su vez, la limitación de no considerar más rango de potenciales que el habitual de corrosión del acero en ese medio $y$, por lo tanto, la incapacidad de predecir comportamientos en otras circunstancias.

Por todo ello, se propone la utilización conjunta y paralela de ambos tipos de medida realizadas sobre aceros embebidos en probetas de mortero $u$ hormigón, que aunque no podrán dar resultados tan fiables como una investigación larga y exhaustiva, puede ser un método suficientemente fidedigno cuando se pretenda conocer, en un relativamente corto espacio de tiempo, la influencia sobre la corrosuión de armaduras de circunstancias capaces de desencadenar ataque, como son: un aditivo del que se desconoce su composición o se sospecha que tenga $\mathrm{Cl}^{-}$, del tamaño de las fisuras en un determinado hormigón, de un determinado tipo de cemento o de un nuevo acero, del contenido en humedad ambiental, de la carbonatación, etc... Así, los $E_{\mathrm{r}}$ deducidos de las curvas galvanostáticas indicarán el peligro latente, o riesgo de corrosión, o susceptibilidad al ataque; y los valores de $R_{\mathrm{p}}$ revelarán la velocidad instantánea de corrosión en las circunstancias exisientes en el momento de efectuar la medida.

\section{A G R A D E C I M I E N T O}

Los autores agradecen al Dpto. de Corrosión y Protección del CENIM y al Dpto. de Química del IETcc las facilidades dadas para la realización del presente trabajo.

\section{B I B L I O G R A F I A}

(1) Andrade C. y Gonzalez, J. A.: Materiales de Const. n. $.^{\circ} 168,4 .^{\circ}$ trimestre de 1977.

(2) KAESCHE H.: Zement-Kalk-Gips 7 (1959) 289.

(3) Baumel A. y Engell H. J.: Archiv. für Eisenhütt. 30, 7 (1959) 417.

(4) Gonzalez J. A. y Andrade C.: Materiales de Const. n..$^{\circ}$ 165, 1..$^{\circ}$ trimestre de 1977.

(5) RC-75: Pliego de prescripciones técnicas generales para la recepción de cementos. MOP 1975. Madrid.

(6) Gonzalez J. A. y Andrade C.: Werkstoffe und Korrosion (en prensa).

(7) Lewis D. A. y Copenhagen W. J.: Corrosión 15, 7 (1959) 382. 